\title{
Spinal manipulation and therapeutic exercises in treating post-surgical resurgent lumbar radiculopathy
}

\author{
Vinicius Tieppo Francio ${ }^{1,2,3, *}$, Chris Towery ${ }^{1,4}$, Saeid Davani ${ }^{1}$ \\ and Tony Brown ${ }^{5}$
}

${ }^{1}$ USAT College of Medicine, University of Science, Arts and Technology, PO Box 506, S. Mayfield Estate Dr., Olveston, Montserrat MSR1350, British West Indies, ${ }^{2}$ Essential Integrative Health, Spine and Pain Management, 3110 SW 89th, suite 200e, Oklahoma City, OK 73159, USA, ${ }^{3}$ Variety Care, Community Health Center, 1025 Straka Terrace, Oklahoma City, OK 73139, USA, ${ }^{4}$ Faces Dermatology, 23206 Lyons Ave Suite 104, Santa Clarita, CA 91321, USA, and ${ }^{5}$ Harvard University, Harvard Summer School, 51 Brattle St, Cambridge, MA 02138-3722, USA

${ }^{*}$ Correspondence address. University of Science, Arts and Technology, USAT College of Medicine, P.O. Box 506, S. Mayfield Estate Dr., Olveston, Montserrat, British West Indies. Tel: +1 (405) 703-7300; Fax: +1 (405) 737-0221; E-mail: vinicius.francio@usat.edu

\begin{abstract}
Lumbar radiculopathy due to disc herniation is one of the most common conditions seen in orthopedic practices worldwide. Surgical intervention is often necessary, and most patients improve without reoccurrences. The purpose of this case is to describe the successful outcome of post-surgical resurgent lumbar radiculopathy treated with spinal manipulation and therapeutic exercises. Here, we discuss the case of a 42-year-old male who received twelve treatments of spinal manipulation, in addition to therapeutic McKenzie methods exercises. By the end of the treatment plan, the patient was asymptomatic and his outcome assessment score (Oswestry questionnaire) showed an $89 \%$ improvement. In a three-month follow-up, he remained stable, and therefore, this case reports a successful outcome and the worthiness of considering spinal manipulation and therapeutic exercises in patients suffering from resurgent lumbar radiculopathy, even when postsurgical changes are present, if there are no absolute contraindications.
\end{abstract}

\section{INTRODUCTION}

Lumbar radiculopathy is commonly caused by degenerative conditions, such as a herniated nucleus pulposus or lumbar spinal stenosis, and often results from neurogenic root inflammation that has progressed enough to cause neurologic symptoms in the areas supplied by the affected nerve root(s) [1]. Lumbar radiculopathy occurs in $\sim 3-5 \%$ of the population, and $10-25 \%$ develop reoccurrence of symptoms that usually persist for more than 6 weeks [2]. The anatomy of the lumbar epidural space is the key to understand the mechanism of lumbar radiculopathy. The sinuvertebral nerve innervates structures in the lumbar epidural space. The nerve originate distal to the dorsal root ganglion, and then returns via the intervertebral foramen to supply the arteries, venous plexus and lymphatics. The same feedback cycle of innervation is found in the outer portion of the intervertebral disc and to the deep dorsal lumbar musculature, such as the multifidus muscles [3]. Pain is mediated by inflammatory mechanisms that involve substances such as phospholipase A2, nitric oxide, and prostaglandin E. These mediators are found in the nucleus pulposus.

Received: February 3, 2017. Revised: June 29, 2017. Accepted: 0, 0

(c) The Author 2017. Published by Oxford University Press.

This is an Open Access article distributed under the terms of the Creative Commons Attribution Non-Commercial License (http://creativecommons.org/ licenses/by-nc/4.0/), which permits non-commercial re-use, distribution, and reproduction in any medium, provided the original work is properly cited. For commercial re-use, please contact journals.permissions@oup.com 
Elevated concentrations of these substances due to injury acts on cell membranes to release arachidonic acid, a precursor to other prostaglandins and leukotrienes, that further advance the inflammatory cascade evoking a feedback cycle of axial lumbar mechanical pain and radicular neurogenic pain. Additionally, leukotriene B4 and thromboxane B2 have direct nociceptive stimulatory roles along the dorsal horn, peripheral nerves and spinal nerve roots [4].

Considering a biomechanical perspective the lumbar intervertebral discs are highly susceptible to injury because they are exposed to tremendous forces, mainly by the magnification of the forces that result from the lever effect of the human erect posture and the biomechanics of the intervertebral disc and adjacent motor units [5]. According to Inoue et al. [5], changes in the material properties of the intervertebral disc and the annulus fibrosus tissue (such as increased intersegmental motion instability, degenerative cascade and biomechanical alterations post-surgery) are also considered as a governing factor affecting the dynamic viscoelastic properties of the entire intervertebral disc. Hence, in patients with altered biomechanical pathways due to prior lumbar laminectomy, intervertebral discs tend to have a great susceptibility to recurrent injury, eliciting the above described cascade of inflammatory cytokines, evoking in this case lumbar radiculitis symptomatology due to the altered biomechanical intervertebral disc dysfunction, related to the architectural changes status post laminectomy. Therefore, the consideration of biomechanical interventions, such as spinal manipulation and therapeutic exercises, as used in this case report is a of worthy reflection, in cases where there is no neurological compromise and the symptomatology is musculoskeletal in nature.

\section{CASE REPORT}

This case details a 42-year-old male who presented to our orthopedic spine clinic with 6 weeks active symptomatology of lower back pain with acute left-sided lumbar radiculopathy non-responsive to over-the-counter medications, muscle relaxants and pain medicines. Prior medical history was significant for a lumbar laminectomy surgery 18 months prior, with an unremarkable recovery free from infection or side effects. He completed therapy post-surgery, successfully regained function and returned to daily living activities. Prior to surgery, the patient had lumbar epidural injections without relief of radicular pain and back pain. Recent clinical exam revealed acute symptoms of lumbar radiculopathy with no motor weakness. Left-sided seated straight leg raiser maneuver was positive, as well as Slump's maneuver for left-sided radicular pain into the lower extremity, below the knee into the left dorsum of the foot. Left-sided femoral nerve stretch test was negative as well as negative Hoffman's sign and right-sided neuro tension maneuvers. Sensation was slightly decreased with light touch along the $\mathrm{L} 5$ dermatome at the dorsum of the left foot. There were no changes to vibratory sense testing bilaterally. Updated lumbar spine MRI (Figs 1 and 2) revealed post-surgical laminectomy morphological changes, with lumbar disc degenerative disease, disc bulging, and neuroforaminal with central canal narrowing. Due to the lack of neurological compromise, there was no immediate need for surgical intervention, therefore conservative treatment with spinal manipulative treatment (SMT) and therapeutic exercises was considered first. Lumbar chemonucleolysis and other spinal injections were discussed, but the patient opted for conservative management first. There were

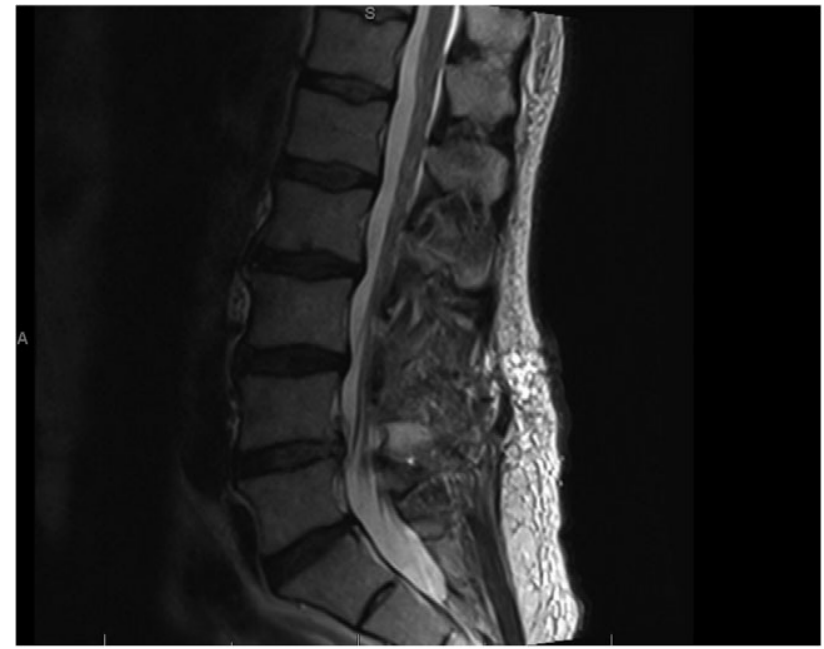

Figure 1: Sagittal lumbar spine MRI with radiological findings of multileve multifactorial lumbar disc disease with bulging disc, and s/p laminectomy at the $\mathrm{L} 4 / 5$ level.

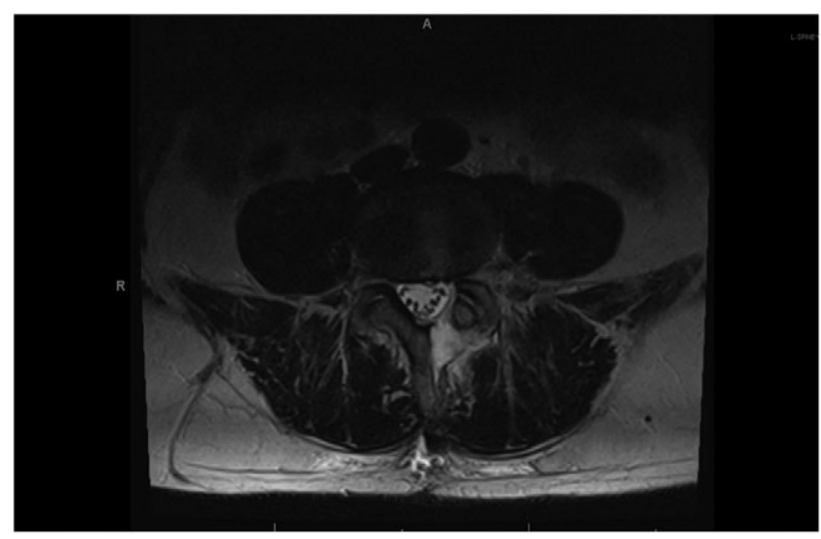

Figure 2: Axial lumbar spine MRI with radiological findings of left neuroforamina narrowing with bulging disc, in addition to residual scar tissue surrounding the left L4/5 neuroforaminal level.

no reasons suggestive of absolute contraindications to SMT, although history of lumbar spine surgery is considered a relative contra indication for spinal manipulation, especially if there is a neurological deficit. Therefore, lacking neurological deficits or absolute contraindications, there was no need for immediate surgery. Spinal manipulation and therapeutic exercise treatment plan was recommended and initiated. The patient consulted with the orthopedic spine surgeon prior to start the treatment intervention, whom acknowledged the lack of neurological deficits, and the trial of spinal manipulation and exercise program as a reasonable intervention, prior to interventional spinal injections or surgery.

The treatment program utilized in this case study emphasized lumbar spinal manipulation performed by a licensed practitioner, in addition to therapeutic exercises education. In this specific case, the treatment program consisted of twice a week for 6-week period in clinic treatment with spinal manipulation, with 12 treatment visits, including training and education on the McKenzie Method of Mechanical Diagnosis and Therapy (MDT) program for lumbar disc radiculopathy to be performed at home on a consistent basis. Treatment protocol 
consisted of high velocity-low amplitude manual spinal manipulation of the lumbar spinal segments treated, L4, L5 and S1 with a lateral decubitus position. Prior to intervention, the patient's Oswestry score was significant for severe lower back musculoskeletal disability (52\%), due to acute lumbar radiculopathy The patient was asymptomatic from the lower back pain, and radicular complaint, at the end of the treatment plan,

The outcome assessment score (Oswestry) showed an $89 \%$ functional musculoskeletal improvement in tolerance to his activities of daily living and work duties. He remained stable with no significant pain or disability score on the Oswestry questionnaire at 3-month follow-up appointment. After the treatment plan, the patient had a consult with the orthopedic spine surgeon, and was discharged with acknowledgment of complete resolution of prior symptoms, and no need for further diagnostic studies or treatment interventions, including spinal surgery.

\section{DISCUSSION}

Recent research [6] demonstrates that the most commonly used intervention in patients with lumbar radiculopathy with significant and progressive motor deficits, cauda equina syndrome with bowel and bladder dysfunction are: discectomy, fusion, chemonucleolysis and percutaneous discectomy. Further guidelines encourage least invasive methods, such as epidural steroid injections, nerve blocks and nerve ablations as a reasonable approach in cases without neurological dysfunction. Outcome success rates of $75.4 \%$ have been reported with the use of epidural and selective nerve blocks [7], yet outcomes are significantly better when these are used in conjunction with oral medications and, in patients who had a herniated lumbar nucleus pulposus and radiculopathy [8]. Spinal manipulation is generally utilized to promote separation between the joint surfaces, enhance smooth joint gliding, promote joint gapping, reduce focal pain by decreasing the release of cytokines and stimulating mechanoreceptors [9]. Spinal manipulation and therapeutic exercises have been shown to be of potential benefits for patients with low-back pain with radiculopathy, even following laminectomy and decompression surgery, as in the present case report [9-11]. Therapeutic exercises (McKenzie methods) suggest the mechanism of adhesions breaking through mechanical lumbar hyperextension, by eliciting dynamic stretching of the muscle spindles and Golgi Tendon Organs, consequently reducing pain and improving musculoskeletal function [11].

Approximately $10 \%$ of low-back pain complaints are accompanied by lumbar radiculopathy. Of those, over $50 \%$ indicate a pattern of intermittent presentation. The most common cause of lumbar radiculopathy is nerve root irritation, often due to intervertebral disc herniation, which is a common reoccurrence in patients with a history of lumbar laminectomy [12]. Clinical management guidelines are unclear on which interventions are most appropriate in patients suffering from recurrent radiculopathy following laminectomy. However, it is well established that if neurological compromise is present, surgery is the option most commonly chosen [13]. Clinical guidelines recommend the use of epidural steroid injections and nerve blocks for short-term symptomatic management of lumbar radiculopathy, and these interventions are also often used in clinical practice to facilitate progression towards rehabilitation methods $[11,14,15]$. Spinal manipulation and therapeutic exercises treatments may be incorporated into the initial treatment of acute lower back pain with radiculopathy to facilitate the patient's transition to active exercise program [16-18]. Superior patient satisfaction levels have been demonstrated among those patients who receive spinal manipulation based care [1].

Furthermore, McKenzie method exercise program is another useful intervention that licensed practitioners may incorporate for patients with lumbar disc injury and radiculopathy $[11,16]$. The proposed mechanisms of action with the combination of spinal manipulation and McKenzie exercise include enhance joint gapping, reduce focal pain by decreasing the release of cytokines and stimulating mechanoreceptors; as well as breaking through adhesions with mechanical lumbar hyperextension, by eliciting dynamic stretching of the muscle spindles and Golgi Tendon Organs, consequently reducing pain and improving musculoskeletal function [9-11, 19-25]. The dynamic stretching produced by spinal manipulation induces a bombardment of activity in joint and muscular mechanoreceptors that is transmitted along largefibers afferents, which produces inhibitory nociceptive effects within the nervous system [22-25].

Recent research [16-19] confirms the worthiness of these treatment interventions in lumbar radiculopathy, which there is specific moderate evidence that these are effective and a viable noninvasive treatment option for patients with acute lumbar spine-related extremity symptoms. Spinal manipulation has been considered very effective [26]; and is reported to reduce the utilization of a second lumbar spine surgery by approximately two-thirds, when used appropriately [27], such as in the present case. The proposed biomechanical changes in the lumbar joint unit when spinal manipulation is applied include decreased intradiscal pressure; increased intervertebral disc space height; and enhanced physiological range of motion of the facet joint [28]. Furthermore, recent studies with MRIs pre and post intervention have demonstrated separation between the joint surfaces (gapping), which tends to improve joint gliding and reduce pain by decreasing the release of cytokines, and stimulating mechanoreceptors [28-30]. The successful outcome of this case is supported by other similar studies $[18,19,31-36]$ of radiculopathy or neurologic symptoms associated to spinal joint dysfunctions, which symptoms improved significantly following spinal manipulation and therapeutic exercises. Thus, in cases were the patient presents with lack of neurological symptoms or complications, but substantial mechanical musculoskeletal dysfunctions, the utilization of spinal manipulation and therapeutic exercises, in an important conservative clinically and cost effective consideration.

Therefore, the present case urges the worthiness of considering spinal manipulation and therapeutic exercises in patients suffering from acute lumbar radiculopathy, even when postsurgical changes are present, if there are no contraindications.

\section{ACKNOWLEDGEMENT}

Written patient consent obtained.

\section{CONFLICT OF INTEREST STATEMENT}

The authors received no benefit in any form, nor will they receive such from a commercial party related directly or indirectly to he subject of this article.

\section{FUNDING}

No funding sources to declare. 


\section{AUTHOR'S CONTRIBUTIONS}

V.T.F. is the primary and corresponding author. C.T., S.D. and T.B. contributed equally to this manuscript.

\section{REFERENCES}

1. Leininger B, Bronfort G, Evans R, Reiter T. Spinal manipulation or mobilization for radiculopathy: a systematic review. Phys Med Rehabil Clin N Am 2011;22:105-25.

2. Casey E. Natural history of radiculopathy. Phys Med Rehabil Clin N Am 2011;22:1-5.

3. Linqiu Z, Schneck CD, Shao Z. The anatomy of dorsal ramus nerves and its implications in lower back pain. Neurosci Med 2012;3:192-201.

4. Omoigui S. The Biochemical Origin of Pain: the origin of all pain is inflammation and the inflammatory response. PART 2 of 3-inflammatory profile of pain syndromes. Med Hypotheses 2007;69:1169-78.

5. Inoue N, Espinoza Orías AA. Biomechanics of intervertebral disc degeneration. Orthop Clin North Am 2011;42: 487-99.

6. Chou R, et al. Surgery for low back pain: a review of the evidence for an American Pain Society Clinical Practice Guideline. Spine (Phila Pa 1976) 2009;34:1094-109.

7. Bhatia A. Transforaminal epidural steroid injections for treating lumbosacral radicular pain from herniated intervertebral discs: a systematic review and meta-analysis. Anesth Analg 2016;122:857-70.

8. Thackeray A, Fritz JM, Brennan GP, Zaman FM, Willick SE. A Pilot Study examining the effectiveness of physical therapy as an adjunct to selective nerve root block in the treatment of lumbar radicular pain from disc herniation: a randomized controlled trial. Phys Ther 2010;90:1717.

9. Reed WR, Liebschner MA, Sozio RS, Pickar JG, Gudavalli MR. Neural response during a mechanically assisted spinal manipualtion in an animal model: a pilot study. $J$ Nov Physiother Phys Rehabil 2015;2:20-7.

10. Clijsters M, Fronzoni F, Jenkins H. Chiropractic treatment approaches for spinal musculoskeletal conditions: a crosssectional survey. Chiropr Man Therap 2014;22:33.

11. Clare H. A systematic review of efficacy of McKenzie therapy for spinal pain. Aust J Physiother 2004;50:209-16.

12. Atlas SJ. The Quebec task force classification for spinal disorders and the severity, treatment, and outcomes of sciatica and lumbar spinal stenosis. Spine (Phila Pa 1976) 1996;21:2885-92.

13. Sharma H, Lee SW, Cole AA. The management of weakness caused by lumbar and lumbosacral nerve root compression. J Bone Joint Surg Br 2012;94:1442-7.

14. Jewell DV, Riddle DL. Interventions that increase or decrease the likelihood of a meaningful improvement in physical health in patients with sciatica. Phys Ther 2005;85:1139-50.

15. Atlas SJ, Keller RB, Wu YA, Deyo RA, Singer DE. Long-term outcomes of surgical and nonsurgical management of sciatica secondary to a lumbar disc herniation: 10 year results from the Maine Lumbar Spine Study. Spine (Phila Pa 1976) 2005;30:927-35.

16. Browder DA, Childs JD, Cleland JA, Fritz JM. Effectiveness of an extension-oriented treatment approach in a subgroup of subjects with low back pain: a randomized clinical trial. Phys Ther 2007;87:1608-18.

17. Schneider MJ, et al. Exploratory analysis of clinical predictors of outcomes of nonsurgical treatment in patients with lumbar spinal stenosis. J Manipulative Physiol Ther 2016 Feb; 39:88-94.

18. Chour R, et al. Nonphamacological therapies for low back pain: a systematic review for an American College of Physician Clinical Practice Guideline. Ann Intern Med 2017; 166:493-505.

19. Dougherty P, Bajwa S, Burke J, Dishman JD. Spinal manipulation postepidural injection for lumbar and cervical radiculopathy: a retrospective case series. J Manipulative Physiol Ther 2004;27:449-56.

20. Pickar JG. Neurophysiological effects of spinal manipulation. Spine J 2002;2:357-71.

21. Pickar JG, Wheeler JD. Response of muscle proprioceptors to spinal manipulative-like loads in the anesthetized cat. J Manipulative Physiol Ther 2001;24:2-11.

22. Savva C, Giakas G, Efstathiou M. The role of the descending inhibitory pain mechanism in musculoskeletal pain following high-velocity, low amplitude thrust manipulation: a review of the literature. J Back Musculoskelet Rehabil 2014;27: 377-82.

23. Ostelo RW, et al. Rehabilitation following first-time lumbar disc surgery: a systematic review within the framework of the Cochrane Collaboration. Spine (Phila Pa 1976) 2003;28: 209-18.

24. Vacek J. Sensory-motor approach to the stabilization system of the spine in patient with chronic back pain International Musculoskeletal Medicine 2012;34:48-50.

25. Bialosky JE, Bishop MD, Price DD, Robinson ME, George SZ. The mechanisms of manual therapy in the treatment of musculoskeletal pain: a comprehensive model. Man Ther 2009;14:531-8.

26. Chou A, et al. Clinical efficacy assessment subcommittee of the American College Of Physicians; American College Of Physicians; American Pain Society low back pain guidelines panel. Diagnosis and treatment of low back pain: a joint clinical practice guideline from the American College of Physicians and the American Pain Society. Ann Intern Med 2007;147:478-91.

27. Rasmussen RA, et al. Rates of lumbar disc surgery before and after implementation of multidisciplinary nonsurgical spine clinics. Spine 2005;30:2469-73.

28. Cramer GD, et al. Magnetic resonance imaging zygapophyseal joint space changes (gapping) in low back pain patients following spinal manipulation and side-posture positioning: a randomized controlled mechanisms trial with blinding. J Manipulative Physiol Ther 2013;36:203-17.

29. Xia $T$, et al. Similar effects of thrust and nonthrust spinal manipulation found in adults with subacute and chronic low back pain: a controlled trial with adaptive allocation. Spine (Phila Pa 1976) 2016;41:E702-9.

30. Cramer GD, et al. Evaluating the relationship among Cavitation, Z Joint Gapping, and spinal manipulation: an exploratory case series. J Manipulative Physiol Ther 2011;34: 2-14.

31. T Francio V. Syringomyelia and Arnold-Chiari malformation associated with neck pain and left arm radiculopathy treated with spinal manipulation. BMJ Case Rep 2014. Nov (9); 2014.

32. T Francio V. Chiropractic care for foot drop due to peroneal nerve neuropathy. J Bodyw Mou Ther 2014;18:200-3.

33. Peterson CK, et al. Symptomatic, magnetic resonance imagingconfirmed cervical disk herniation patients: a comparativeeffectiveness prospective observational study of 2 age- and 
sex-matched cohorts treated with either imaging-guided indirect cervical nerve root injections or spinal manipulative therapy. J Manipulative Physiol Ther 2016;39:210-7.

34. Gudavalli MR, Olding K, Joachim G, Cox JM. Chiropractic distraction spinal manipulation on postsurgical continued low back and radicular pain patients: a retrospective case series. J Chiropr Med 2016;15:121-8.
35. Francio VT, Boesch R, Tunning M. Treatment of a patient with posterior cortical atrophy (PCA) with chiropractic manipulation and Dynamic Neuromuscular Stabilization (DNS): a case report. J Can Chiropr Assoc 2015;59:37-45.

36. Alagha B. Chiropractic and rehabilitation management of a patient with extraforaminal entrapment of L4 nerve with balance problem. J Back Musculoskelet Rehabil 2015;28:603-7. 